\title{
Ziele, Fragestellungen und Hypothesen
}

Aufbauend auf den dargelegten theoretischen Modellen und der empirischen Befundlage zielt diese Arbeit mit den folgenden vier empirischen Studien im Zentrum darauf ab, das Verständnis und die Bedeutung von Heterogenität im Kontext beruflicher Orientierung Jugendlicher als Basis bedarfsorientierter Unterstützung in Schule zu stärken.

Verschiedene theoretische Konzepte (Boudon, 1974; Bourdieu, 1982; Gottfredson, 1981) weisen auf eine heterogene Ausgangslage der Jugendlichen in ihrer beruflichen Entwicklung hin. Die daraus entstehenden ungleichen Chancen im Bildungssystem und die Unterschiede in den Bildungs- und Berufsverläufen wurden vielfach empirisch nachgezeichnet (Beicht \& Walden, 2019; Heublein \& Schmelzer, 2018; OECD, 2014; Steinritz et al., 2016). Darüber hinaus resultieren heterogene Unterstützungsbedarfe aus den differierenden Herausforderungen, die Jugendliche in unterschiedlichen Phasen ihrer beruflichen Orientierung bewältigen müssen (Driesel-Lange et al., 2020; Herzog et al., 2006). Schließlich weist die aktuelle Befundlage darauf hin, dass Jugendliche in ihrer Entwicklung berufswahlrelevanter Kompetenzen unterschiedlich weit fortgeschritten sind (DrieselLange \& Kracke, 2017). Nichtsdestotrotz erfolgt die Unterstützung beruflicher Orientierung im schulischen Kontext mehrheitlich ohne eine Bezugnahme auf die sich ergebenden individuellen Bedarfe der Einzelnen. Zentrale Herausforderungen bei der Qualitätssicherung und Innovation beruflicher Orientierung stellen neben personeller und finanzieller Ressourcenknappheit die Informationsdefizite hinsichtlich der Effekte von berufsorientierenden Maßnahmen sowie Hindernisse in der Diagnostik individueller Bedarfe dar (Ohlemann et al., 2016).

Ausgehend von den dargestellten theoretischen Betrachtungen und empirischen Befunden analysiert diese Arbeit daher die Heterogenität beruflicher Entwicklung Jugendlicher in ihrer Multidimensionalität. Die Betrachtung erfolgt 
insbesondere unter Einbezug demografischer, leistungs- und entwicklungsbezogener Aspekte, um individuelle Bedarfe zu spezifizieren, Jugendliche mit ähnlichen Bedarfen zu identifizieren und schließlich auf Basis dieser Profilbildung eine Binnendifferenzierung beruflicher Orientierung im schulischen Kontext zu ermöglichen.

Der besondere Beitrag dieser Arbeit liegt dabei in der systematischen, theoriegeleiteten und empirisch fundierten Herleitung der heterogenen Unterstützungsbedarfe Heranwachsender in ihrer beruflichen Entwicklung. Dafür wurden in dieser Arbeit in einem ersten Schritt die relevanten Theorien und Modelle entfaltet, zueinander in Bezug gestellt und mit der entsprechenden statistischen Datenlage und der bestehenden Forschungsliteratur verknüpft. In einem zweiten Schritt werden die identifizierten Forschungsdesiderate in vier empirischen Studien aufgegriffen. Folglich besteht der zweite, besonders hervorzuhebende Beitrag dieser Arbeit in seiner mehrdimensionalen empirischen Erfassung und Vergegenständlichung individueller Bedürfnisse Jugendlicher. Beide Aspekte, sowohl die systematische Herleitung der heterogenen Bedarfe als auch ihre empirische Analyse, bieten Ansatzpunkte zur Rückkopplung in die Theoriebildung und die empirische Forschung. Des Weiteren ermöglichen die theoriegeleiteten, empirischen Analysen der vorgelegten Arbeit durch die Formulierung handlungsbezogener Konsequenzen den von Lent (2017) geforderten Theorie-Empirie-Praxistransfer.

Vier Studien beleuchten unterschiedliche Perspektiven der Fragestellung. Die Studie in Kapitel 9 befasst sich mit den Zusammenhängen zwischen berufsorientierenden Maßnahmen und der durch die Jugendlichen subjektiv wahrgenommenen beruflichen Orientierung. Dabei interessiert zum einen die Frage, inwiefern Schüler*innen in Abhängigkeit ihres Geschlechts, ihrer Muttersprache und ihres schulischen Leistungsstands unterschiedlich stark von verschiedenen Maßnahmen profitieren. Zum anderen greift die Studie das Forschungsdesiderat der intersektionalen Betrachtung individueller Merkmale auf und beleuchtet ihre differierenden Effekte auf die berufliche Orientierung Jugendlicher. Basierend auf den in Kapitel 3 vorgestellten Theorien sowie der dargelegten empirischen Befundlage wird die Frage nach den Zusammenhängen zwischen den persönlichen Merkmalen Jugendlicher und den von ihnen besuchten berufsorientierenden Maßnahmen in Bezug auf den subjektiven Stand der beruflichen Orientierung wie folgt ausdifferenziert:

(1) Stehen die persönlichen Merkmale (Geschlecht, Muttersprache und Schulleistung) mit dem subjektiven Stand der beruflichen Orientierung in Zusammenhang? 
(2) Welchen Effekt haben Maßnahmen beruflicher Orientierung auf die durch die Jugendlichen empfundene berufliche Orientierung?

(3) Beurteilen Jugendliche aufgrund unterschiedlicher persönlicher Merkmale ihre berufliche Orientierung unterschiedlich stark in Abhängigkeit der Teilnahme an den betrachteten Maßnahmen beruflicher Orientierung?

Die Studie in Kapitel 10 befasst sich mit der Konstruktion eines zentralen Berufswahlkompetenz-Kernwerts als Instrument schulinterner Berufswahlkompetenzdiagnostik. Im Zentrum der explorativen Studie stehen folgende Fragestellungen:

(1) Inwiefern ist die Konstruktion eines einzelnen komplexitätsreduzierenden Normwerts zur Diagnostik von Berufswahlkompetenz möglich?

(2) Lässt sich der Entwicklungsstand einer einzelnen Berufswahlkompetenzfacette anhand des Berufswahlkompetenz-Kernwerts ableiten?

Während die ersten beiden Studien auf variablenzentrierten Analyseverfahren basieren, verwenden die beiden folgenden Studien personenzentrierte Auswertungsmethoden. Die in Kapitel 11 erläuterte Studie beleuchtet wieder den Aspekt der Berufswahlkompetenzentwicklung als Determinante zur Individualisierung bzw. Binnendifferenzierung. Jedoch erfolgt die Einteilung der Jugendlichen im Gegensatz zur Studie in Kapitel 10 über die Ausdifferenzierung der einzelnen Berufswahlkompetenzfacetten. Mittels des Verfahrens der latenten Profilanalyse (vgl. Teilkapitel 8.2) können homogene Gruppen mit ähnlichen Profilen innerhalb einer sonst heterogenen Schülerschaft identifiziert werden (Nylund, 2007).

Basierend auf der in Kapitel 11 vorgestellten Befundlage wird Folgendes angenommen:

(1) Es existieren Berufswahlkompetenzprofile, die sowohl qualitativ als auch statistisch voneinander differenzierbar sind.

(2) Die Profile folgen einem ähnlichen Muster hinsichtlich der Ausprägung der einzelnen Berufswahlkompetenzfacetten, unterscheiden sich aber in ihrem Gesamtniveau.

(3) Es bestehen keine statistisch signifikanten Unterschiede in der Altersverteilung zwischen den Profilgruppen.

(4) Die Verteilung der Mädchen und Jungen in den Profilgruppen unterscheidet sich statistisch signifikant.

(5) Die Existenz konkreter Berufswünsche beeinflusst die Profilgruppenzugehörigkeit. 
Die in Kapitel 12 veranschaulichte Studie befasst sich mit den zeitlichen Mustern des Berufswahlkompetenzerwerbs. Unter der Annahme individueller, diskontinuierlicher Entwicklungsprozesse (vgl. Teilkapitel 6.1) greift die Arbeit auf das in diesem Forschungsfeld eher selten genutzte Verfahren der latenten Transitionsanalyse zurück. Wie in Teilkapitel 8.2 beschrieben wird, erlaubt die latente Transitionsanalyse die Betrachtung eines zeitlichen Verlaufs als Abfolge mehrerer, zeitlich aufeinander folgender Phasen. Aufbauend auf der Befundlage, die in Abschnitt 12.2 dargestellt ist, wird die explorative Untersuchung von Verlaufsprofilen im individuellen Berufswahlkompetenzerwerb wie folgt strukturiert:

(1) Der Berufswahlkompetenzerwerb von Schüler*innen variiert zeitlich und niveaubezogen und lässt sich anhand unterschiedlicher Bewegungsmuster darstellen.

(2) Im Berufswahlprozess wechseln die Schüler*innen zwischen den vier in Kapitel 11 identifizierten Profilgruppen der Berufswahlkompetenz.

(3) Alle Schüler*innen erleben über die beobachtete Zeit eine positive Entwicklung ihrer Berufswahlkompetenz.

Die empirischen Studien (Kapitel 9,10,11 und 12) sind eingerahmt von einer einleitenden Darstellung der untersuchten Daten (Abschnitt 8.1) und der verwendeten statistischen Auswertungsmethoden (Abschnitt 8.2) sowie einer abschließenden Gesamtdiskussion in Kapitel 13. Die Ergebnisse werden darin in Bezug zu dem theoretischen Modell der Berufswahlkompetenz von Driesel-Lange et al. (2010) gesetzt und in den aktuellen Forschungsstand eingeordnet. Die Gesamtdiskussion erörtert ferner die Grenzen der empirischen Befunde und entfaltet die sich aus den Ergebnissen neu ergebenden, weiterführenden Forschungsdesiderata. Abschließend werden die Implikationen der empirischen Befunde für die pädagogische Praxis in der Unterstützung beruflicher Orientierung diskutiert. 
Open Access Dieses Kapitel wird unter der Creative Commons Namensnennung 4.0 International Lizenz (http://creativecommons.org/licenses/by/4.0/deed.de) veröffentlicht, welche die Nutzung, Vervielfältigung, Bearbeitung, Verbreitung und Wiedergabe in jeglichem Medium und Format erlaubt, sofern Sie den/die ursprünglichen Autor(en) und die Quelle ordnungsgemäß nennen, einen Link zur Creative Commons Lizenz beifügen und angeben, ob Änderungen vorgenommen wurden.

Die in diesem Kapitel enthaltenen Bilder und sonstiges Drittmaterial unterliegen ebenfalls der genannten Creative Commons Lizenz, sofern sich aus der Abbildungslegende nichts anderes ergibt. Sofern das betreffende Material nicht unter der genannten Creative Commons Lizenz steht und die betreffende Handlung nicht nach gesetzlichen Vorschriften erlaubt ist, ist für die oben aufgeführten Weiterverwendungen des Materials die Einwilligung des jeweiligen Rechteinhabers einzuholen. 